\title{
Bacteriological Profile and Antimicrobial Susceptibility Pattern of Indian Currency Circulating in a Tertiary Care Hospital of South India
}

\author{
Zarrin Afroz* \\ Department of Microbiology, G.S Medical College and Hospital, Pilkhuwa-245304 \\ District-Hapur, Uttar Pradesh, India \\ *Corresponding author
}

\begin{tabular}{|c|}
\hline Keywords \\
\hline $\begin{array}{l}\text { Currency notes, } \\
\text { Bacteriological } \\
\text { profile, Antibiotic } \\
\text { susceptibility pattern }\end{array}$ \\
\hline Article Info \\
\hline $\begin{array}{l}\text { Accepted: } \\
\text { 16 March } 2018 \\
\text { Available Online: } \\
10 \text { April } 2018\end{array}$ \\
\hline
\end{tabular}

\section{A B S T R A C T}

Currency in hospital is commonly contaminated with different pathogenic and potential pathogenic organisms and all people handling currency are invariably exposed to these microorganisms. To find the profile of bacteria on Indian currency and the antibiotic susceptibility pattern of the isolated bacteria. It was a prospective study from September 2014 to February 2015.A total of 200 currency notes of different denominations, taken from OPD registration counters, hospital pharmacy, laboratory technicians, doctors, nurses and other hospital staffs were analysed. Among the 200 currency notes of five different denominations $172(86 \%)$ were found to be contaminated and $28(14 \%)$ were sterile. A total of 214organisms were isolated from 172 contaminated currency notes. Among the 214 isolates $106(49.53 \%)$ were pathogenic and $108(50.47 \%)$ were non-pathogenic organisms. Out of 214 isolates the percentage of Gram positive isolates were 186 (86.9\%) and $28(13.1 \%)$ were Gram negative bacilli. Among Gram positive cocci the most common isolate was Enterococcus 54 (25.2\%) followed by CONS 48 (22.4\%) and micrococci 30 (14.1\%). Among Gram negative bacilli the most common isolate was Pseudomonas aeruginosa $14(6.6 \%)$ followed by Escherichia coli 10 (4.7\%). Among the currency notes of different denominations most of the organisms were present on 10 rupees note and 20 rupees note $50(23.4 \%)$ followed by 50 rupees note $46(21.5 \%)$. Minimum no of organisms were present on 500 rupees note $32(14.9 \%)$. Among Gram positive isolates maximum susceptibility was seen against vancomycin and linezolid $126(100 \%)$. Among the Gram negative isolates maximum susceptibility was seen against imipenem $28(100 \%)$ followed by piperacillin - tazobactam $26(92.8 \%)$. With the knowledge of the likely organisms isolated from currency notes and their resistance pattern, good money handling practices such as washing hands with soap and water before eating and avoiding using saliva during counting money can reduce risk of infection.

\section{Introduction}

Currency is handled by all categories of people and may be contaminated during coughing, sneezing, touching with hands and placement on dirty surfaces. Many people tongue-wet their fingers when counting money and contaminate their fingers as well as currency notes. So, it is obvious that gets on hands may be transferred to money and viceversa (Pal et al., 2013). The environment plays a critical role in transmission to humans, with 
many environmental materials serving as vehicles. Microbial contaminants may be transmitted either directly, through hand- tohand contact, or indirectly, via food or other inanimate objects like fomites. Paper currency offers a larger surface area as a breeding ground for pathogens. Microbes may persist on it for longer periods (Rote et al., 2010; AlGhamdi et al., 2011).

The isolates include Bacillus cereus, Staphylococcus aureus, Coagulase negative staphylococcus, Klebsiella pneumoniae, Escherichia coli. Bacillus cereus and staphylococcus are observed to be isolated from maximum number of currency notes (Tagoe et al., 2011).

Bacteria are observed to be susceptible to ceftriaxone, gentamicin, norfloxacin and ofloxacin and vancomycin. Susceptibility to amoxicillin, penicillin, ampicillin and cotrimoxazole has been observed to be low (Akoachere et al., 2014).

Contaminated fomites or surfaces play a key role in spread of bacterial infections with antimicrobial resistance. Antimicrobial resistance is a global phenomenon that has resulted in high morbidity and mortality as a result of treatment failures.

Studies of contamination of money with microbial agents is lacking in most developing countries. Shortage of information may contribute to the absence of public health policies regarding currency usage, handling and circulation.

Therefore the current study was designed to add to the limited body of literature on bacterial contamination of currency notes and the antimicrobial susceptibility pattern of the isolates. This will emphasizes the risks associated with microbial contamination on currency notes while handling money.

\section{Materials and Methods}

It was a prospective study from September 2014 to February 2015 which included a total of 200 currency notes of different denominations, taken from OPD registration counters, hospital pharmacy, laboratory technicians, doctors, nurses and other hospital staffs working in BLDEA'S Shri B.M. Patil Medical College Hospital and Research Centre, Vijayapura.

\section{Inclusion criteria}

Single and mixed growth isolated from was included in the study.

\section{Exclusion criteria}

Anaerobic and fungal organisms were excluded from the study.

Currency of 10, 20, 50, 100 and 500 (forty each) were collected randomly without any special selection criteria. Persons handling the money were asked to deposit the currency in a sterile polythene bag. Samples collected were sealed, tagged and taken to the bacteriology laboratory for analysis. A sterile cotton swab soaked in normal saline was rubbed thoroughly on both surfaces of currency notes. Swabs were inoculated on nutrient agar, MacConkey agar and blood agar plates. Nutrient agar and MacConkey agar plates were incubated aerobically and blood agar plates were incubated in the presence of $5 \%$ $\mathrm{CO}_{2}$ at $37{ }^{\circ} \mathrm{C}$ overnight. The isolates were identified by gram staining, colony morphology and standard biochemical tests :catalase, slide and tube coagulase, oxidase, esculin hydrolysis, indole production, methyl red test, Voges-Proskauer test, citrate utilization (IMViC tests), $\mathrm{H}_{2} \mathrm{~S}$ production, ornithine decarboxylase, arginine hydrolase, lysine decarboxylase, urease, nitrate reduction and sugar fermentation tests (Collee and Marr, 
2012). Antibiotic susceptibility tests were done on these isolates by standard Disc diffusion method according to CLSI guidelines. The following antibiotics were tested: For Gram positive cocci penicillin, azithromycin $(15 \mu \mathrm{g})$, cotrimoxazole $(25 \mu \mathrm{g})$, linezolid $(30 \mu \mathrm{g})$, tetracycline $(30 \mu \mathrm{g})$, ciprofloxacin $(5 \mu \mathrm{g})$, gentamicin $(10 \mu \mathrm{g})$,

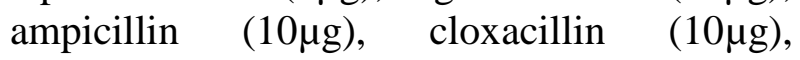
cefuroxime $(30 \mu \mathrm{g})$ and vancomycin $(30 \mu \mathrm{g})$. For Gram negative bacilli ampicillin $(10 \mu \mathrm{g})$, gentamicin $(10 \mu \mathrm{g})$, amikacin $(30 \mu \mathrm{g})$, piperacillin-tazobactam $\quad(100 / 10 \mu \mathrm{g})$, cefuroxime $(30 \mu \mathrm{g})$, cephalexin $(30 \mu \mathrm{g})$, ceftriaxone $(30 \mu \mathrm{g})$, cotrimoxazole $(25 \mu \mathrm{g})$, ceftazidime $(30 \mu \mathrm{g})$, tetracycline $(30 \mu \mathrm{g})$, ciprofloxacin $(5 \mu \mathrm{g})$ and imipenam $(10 \mu \mathrm{g})$.

\section{Results and Discussion}

Among the 200 currency notes of five different denominations $172(86 \%)$ were found to be contaminated and $28(14 \%)$ were sterile. The percentage of contaminated and sterile notes is given in (Table 1).

A total of 214 organisms were isolated from 172 contaminated currency notes. Among the 214 isolates $106(49.53 \%)$ were pathogenic and $108(50.47 \%)$ were non-pathogenic organisms. Non-pathogenic organisms included Coagulase negative Staphylococcus (CONS) 48 (22.4\%) followed by micrococci $30(14.1 \%)$ and Gram positive bacilli 30 (14.1\%) Pathogenic organisms can cause disease in healthy host while non-pathogenic organisms can cause disease in immunocompromised host.

Out of 214 isolates the percentage of Gram positive isolates were $186(86.9 \%)$ and $28(13.1 \%)$ were Gram negative bacilli. Among Gram positive isolates 156 (72.9\%) were Gram positive cocci and $30(14.1 \%)$ were Gram positive bacilli. Among Gram positive cocci the most common isolate was Enterococcus 54 (25.2\%) followed by CONS
48 (22.4\%) and micrococci 30 (14.1\%). Among Gram negative bacilli the most common isolate was Pseudomonas aeruginosa 14 (6.6\%) followed by Escherichia coli $10(4.7 \%)$ and Klebsiella species 4 (1.9\%). Overall Enterococcus was the major organism isolated $54(25.2 \%)$. The distribution of organisms is given in (Table 2).

Among the currency notes of different denominations most of the organisms were present on 10 rupees note and 20 rupees note $50(23.4 \%)$ followed by 50 rupees note 46 $(21.5 \%)$.

Minimum no of organisms were present on 500 rupees note $32(14.9 \%)$. The distribution of organisms on currency notes is given in (Table 3).

\section{Antimicrobial susceptibility pattern of Gram positive organisms}

Among Gram positive cocci antimicrobial susceptibility testing was performed for pathogenic isolates and Coagulase negative Staphylococcus. Maximum susceptibility was seen against vancomycin and linezolid $126(100 \%)$ followed by tetracycline 102 (80.9\%) and ciprofloxacin 98 (77.7\%). Minimum susceptibility was seen against Penicillin $62(49.2 \%)$ followed by ampicillin 64 (50.8\%). Other effective drugs were cotrimoxazole 96 (76.2\%), Gentamicin 94 (74.6\%), azithromycin and cefuroxime 92 (73.1\%).The susceptibility pattern of Gram positive isolates is given in table 4 and figure 1.

\section{Antimicrobial susceptibility pattern of Gram negative organisms}

Among the Gram negative isolates maximum susceptibility was seen against imipenem 28 (100\%) followed by piperacillin - tazobactam $26(92.8 \%)$ followed by amikacin $24(85.7 \%)$. 
Int.J.Curr.Microbiol.App.Sci (2018) 7(4): 1828-1834

Table.1 Percentage of contaminated and sterile notes

\begin{tabular}{|l|l|l|l|l|l|l|}
\hline Currency & Rs 10 & Rs 20 & Rs 50 & Rs 100 & Rs 500 & Total no (\%) \\
\hline Contaminated & 38 & 36 & 36 & 34 & 28 & $\mathbf{1 7 2}(\mathbf{8 6 \%})$ \\
\hline Sterile & 2 & 4 & 4 & 6 & 12 & $\mathbf{2 8}(\mathbf{1 4 \%})$ \\
\hline
\end{tabular}

Table.2 Percentage of organisms isolated from currency notes

\begin{tabular}{|l|l|}
\hline Organisms & No (\%) \\
\hline Enterococcus species & $54(25.2 \%)$ \\
\hline CONS & $48(22.4 \%)$ \\
\hline Micrococci & $30(14.1 \%)$ \\
\hline Gram positive bacilli & $30(14.1 \%)$ \\
\hline Staphylococcus aureus & $24(11.2 \%)$ \\
\hline Pseudomonas aeruginosa & $14(6.5 \%)$ \\
\hline Escherichia coli & $8(4.7 \%)$ \\
\hline Klebsiella species & $4(1.9 \%)$ \\
\hline Total & $\mathbf{2 1 4}$ \\
\hline
\end{tabular}

Table.3 Distribution of organisms on currency notes of different denominations

\begin{tabular}{|l|l|l|l|l|l|l|}
\hline Currency denomination & Rs $\mathbf{1 0}$ & Rs 20 & Rs 50 & Rs 100 & Rs 500 & Total \\
\hline No of currency & 40 & 40 & 40 & 40 & 40 & \\
\hline Enterococcus sp & 12 & 12 & 14 & 8 & 8 & 54 \\
\hline CONS & 10 & 14 & 12 & 6 & 6 & 48 \\
\hline Micrococci & 8 & 10 & 2 & 6 & 4 & 30 \\
\hline Gram positive bacilli & 6 & 6 & 8 & 6 & 4 & 30 \\
\hline Stajphylococcus aureus & 6 & 4 & 4 & 4 & 6 & 24 \\
\hline Pseudomonas aeruginosa & 4 & 2 & 2 & 4 & 2 & 14 \\
\hline Escherichia coli & 4 & - & 4 & 2 & - & 10 \\
\hline Klebsiella species & - & 2 & 2 & - & - & 4 \\
\hline Total no (\%) & $50(23.4 \%)$ & $50(23.4 \%)$ & $46(21.5 \%)$ & $36(16.8 \%)$ & $32(14.9 \%)$ & 214 \\
\hline
\end{tabular}

Table.4 Antimicrobial susceptibility pattern of Gram positive isolates

\begin{tabular}{|l|}
\hline Antimicrobial agent \\
\hline Penicillin \\
\hline Azithromycin \\
\hline Cotrimoxazole \\
\hline Linezolid \\
\hline Tetracycline \\
\hline Ciprofloxacin \\
\hline Gentamicin \\
\hline Ampicillin \\
\hline Cloxacillin \\
\hline Cefuroxime \\
\hline Vancomycin
\end{tabular}

\begin{tabular}{|l|l|l|}
\hline Enterococcus sp & CONS & S.aureus \\
\hline 30 & 26 & 6 \\
\hline 40 & 36 & 16 \\
\hline 40 & 40 & 16 \\
\hline 54 & 48 & 24 \\
\hline 42 & 40 & 20 \\
\hline 44 & 38 & 16 \\
\hline 40 & 38 & 16 \\
\hline 30 & 28 & 6 \\
\hline 38 & 36 & 16 \\
\hline 40 & 32 & 20 \\
\hline 54 & 48 & 24 \\
\hline
\end{tabular}

Total no $(\%)$

$62(49.2 \%)$

$92(73.1 \%)$

$96(76.2 \%)$

$126(100 \%)$

$102(80.9 \%)$

98 (77.7\%)

$94(74.6 \%)$

$64(50.8 \%)$

$90(71.4 \%)$

$92(73.1 \%)$

$126(100 \%)$ 
Table.5 Antimicrobial susceptibility pattern of Gram negative isolates

\begin{tabular}{|l|c|c|c|l|}
\hline Antimicrobial agent & P.aeruginosa & E.coli & Klebsiella sp & Total no (\%) \\
\hline Ampicillin & 2 & 0 & 0 & $\mathbf{2}(\mathbf{7 . 1 4 \% )}$ \\
\hline Gentamicin & 10 & 6 & 4 & $\mathbf{2 0}(\mathbf{7 1 . 4 \% )}$ \\
\hline Amikacin & 12 & 8 & 4 & $\mathbf{2 4}(\mathbf{8 5 . 7 \% )}$ \\
\hline Cefuroxime & 6 & 4 & 2 & $\mathbf{1 2}(\mathbf{4 2 . 8 \% )})$ \\
\hline Cephalexin & 6 & 4 & 2 & $\mathbf{1 2}(\mathbf{4 2 . 8 \% )}$ \\
\hline Ceftriaxone & 8 & 6 & 2 & $\mathbf{1 6} \mathbf{5 7 . 1 \% )}$ \\
\hline Ceftazidime & 10 & 6 & 4 & $\mathbf{2 0}(\mathbf{7 1 . 4 \% )}$ \\
\hline Cotrimoxazole & 10 & 8 & 2 & $\mathbf{2 0}(\mathbf{7 1 . 4 \% )}$ \\
\hline Tetracycline & 10 & 8 & 4 & $\mathbf{2 2}(\mathbf{7 8 . 6 \% )}$ \\
\hline Ciprofloxacin & 10 & 8 & 2 & $\mathbf{2 0}(\mathbf{7 1 . 4 \%})$ \\
\hline P+T & 12 & 10 & 4 & $\mathbf{2 6}(\mathbf{9 2 . 8 \% )}$ \\
\hline Imipenem & 14 & 10 & 4 & $\mathbf{2 8}(\mathbf{1 0 0 \%})$ \\
\hline
\end{tabular}

Fig.1 Antimicrobial susceptibility pattern of Gram positive isolates

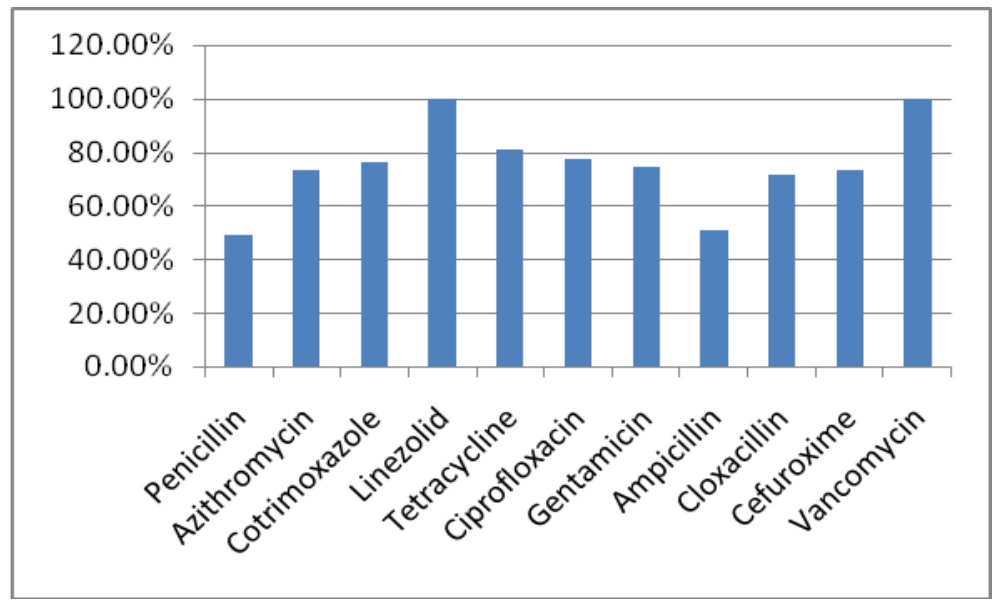

Fig.2 Antimicrobial susceptibility pattern of Gram negative isolates

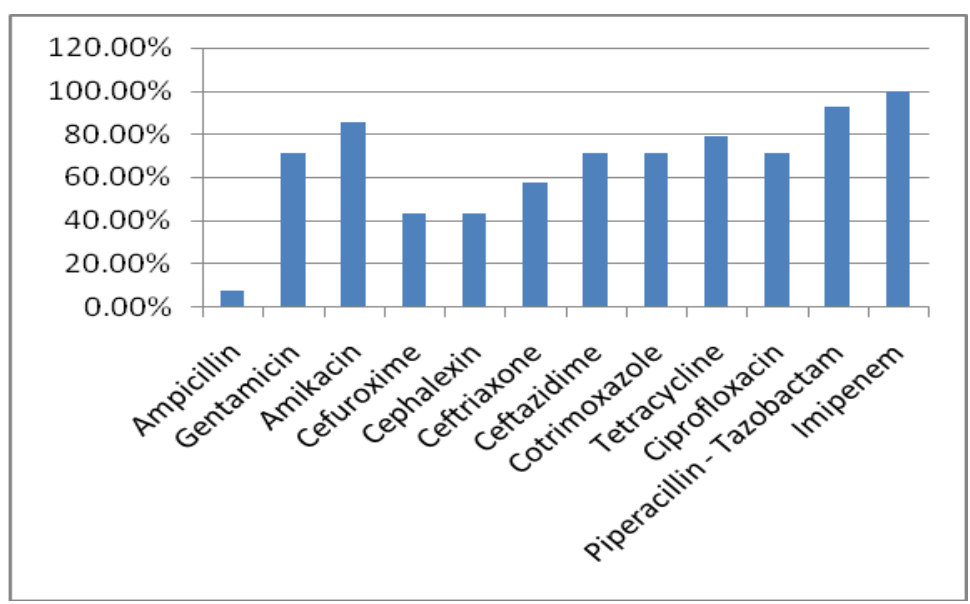


Minimum susceptibility was seen against ampicillin 2 (7.14\%) followed by cephalosporins like cefuroxime and cephalexin 12 (42.8\%).Other effective drugs were tetracycline $22 \quad(78.6 \%)$ followed by ciprofloxacin, gentamicin, cotrimoxazole and ceftazidime $20(71.4 \%)$. The antimicrobial susceptibility pattern of Gram negative isolates is given in table 5 and figure 2.

In day to day use paper currency notes are transferred from one individual to another which transmits the microorganisms present on paper currency notes. In India even paper currency notes are poorly-handled. The major population of India does not carry money in wallets and squeezing of currency notes is a common occurrence (Sadavarte et al., 2014). In the present study among the 200 currency notes of five different denominations 172 (86\%) were found to be contaminated and 28 (14\%) were sterile which is similar to other studies (Pal et al., 2013; Sadavarte et al., 2014).

Among the 214 isolates 106 (49.53\%) were pathogenic and $108(50.47 \%)$ were nonpathogenic organisms. Out of 214 isolates the percentage of Gram positive isolates were 186 (86.9\%) and 28 (13.1\%) were Gram negative bacilli. This is similar to the study conducted by (Pal et al., 2013) where $95 \%$ of the isolates were Gram positive but is in contrast with the study conducted by (Singh et al., 2015) where majority of the pathogenic isolates were Gram negative.

Among Gram positive cocci the most common isolate was Enterococcus 54 (25.2\%) followed by CONS $48(22.4 \%)$ and micrococci 30 $(14.1 \%)$.This is in accordance with the study conducted by (Pavani and Srividya, 2014) but is in contrast with the study conducted by (Mukhopadhyay et al., 2015) where Staphylococcus was the most common Gram positive isolate.

Among Gram negative bacilli the most common isolate was Pseudomonas aeruginosa 14(6.6\%) followed by Escherichia coli 10 (4.7\%) and
Klebsiella species $4(1.9 \%)$. This is in accordance with the study conducted by Mukhopadhyay et al., (2015) but is in contrast with the study conducted by (Pal K et al., 2013; Singh S et al., 2015) where Escherichia coli was the most common Gram negative isolate. This discrepancy in bacterial pattern may be attributed to regional variation of bacterial profile, habits of the local people and also due to the fact that all the Indian studies done so far involve community set up and were not centered on hospitals. Presence of Enterococci is suggestive of significant fecal contamination of currency, and is a reflection of poor local environmental sanitation and personal hygiene. It also signifies a potential minefield for nosocomial infections.

Among the currency notes of different denominations most of the organisms were present on 10 rupees note and 20 rupees note 50 (23.4\%) followed by 50 rupees note $46(21.5 \%)$. Minimum no of organisms were present on 500 rupees note 32 (14.9\%). The lower denomination currency notes are frequently circulated for daily activities where notes get tattered and dirty, therefore become more contaminated. Currency notes of both lower and higher denomination which were in poor conditions were more contaminated than notes those which were in good condition (Sadavarte et al., 2014).

For Gram positive isolates maximum susceptibility was seen against vancomycin and linezolid 126(100\%) followed by tetracycline $102(80.9 \%)$ and ciprofloxacin 98 (77.7\%). Minimum susceptibility was seen against Penicillin 62 (49.2\%) followed by ampicillin 64 $(50.8 \%)$. This pattern is similar to the study conducted by (Pavani and Srividya, 2014).

For Gram negative isolates maximum susceptibility was seen against imipenem $28(100 \%)$ followed by piperacillin tazobactam 26 (92.8\%) and amikacin 24 $(85.7 \%)$. Minimum susceptibility was seen against ampicillin 2 (7.14\%) followed by cephalosporins like cefuroxime and cephalexin 
$12(42.8 \%)$. This pattern is in accordance with the study conducted by (Akoachere et al., 2014). The isolation of bacterial agents including drug resistant organisms from currency notes confirms that currency might be a vector playing an important role in the transmission of pathogenic microorganisms, as well as in the spread of drugresistant strains in the community.

In the present study among the organisms isolated from currency notes the most common Gram positive cocci was Enterococcus followed by CONS. Among Gram negative bacilli the most common isolate was Pseudomonas aeruginosa followed by Escherichia coli. For Gram positive isolates maximum susceptibility was seen against vancomycin and linezolid. For Gram negative bacilli maximum susceptibility was seen against imipenem followed by piperacillin - tazobactam. It can be concluded that currency in hospital is commonly contaminated with different pathogenic and potential pathogenic bacteria and all people handling currency are invariably exposed to these microorganisms. So awareness related to improvement of personal hygiene and good handling practices such as washing hands with soap and water before eating and avoiding using saliva during counting money are strongly recommended as the main pillar to reduce risk of infection.

\section{References}

Akoachere JTK, Gaelle N, Dilonge HM, Akenji TK. 2014. Public health implications of contamination of France CFA (XAF) circulating in Buea (Cameroon) with drug resistant pathogens. BMC research notes, $7: 1-13$.

Al-Ghamdi AK, Abdelmalek SMA, Bamaga MS, Azhar El, Wakid MH, Alsaied Z. 2011. Bacterial contamination of Saudi "one" riyal paper notes. Southeast Asian J Trop Med Public Health, 42:711-16.
Performance Standards for Antimicrobial Disc Susceptibility Tests; Approved standard $11^{\text {th }}$ edition. CLSI document M02A11. Wayne, PA.

Collee JG, Fraser AG, Marmion BP, Simmons A.2012. Mackie and McCartney's Practical Medical Microbiology. 14th edition. New York: Churchill Livingstone.

Mukhopadhyay M, Podder S, Bhattacharya S. 2015. Microbial contamination of Indian currency notes and coins in Kolkata, West Bengal - A Survey. Int. J. Sci. Res., 4:61820.

Pal K, Das NS, Bhattacharya S.2013.Bacteriological profile of Indian currency in a tertiary care hospital in rural Bengal. IJRRMS, 3:23-7.

Pavani G, Srividya Y.2014. Bacterial analysis of currency in circulation in a rural teaching hospital in India. J. Microbiol. Biotech. Res., 4: 34-38.

Rote RB, Deogade NG, Kawale M.2010. Isolation, characterization and antibiotic sensitivity of organism from Indian currency. Asiatic J Biotechnol. Resources, 03:255-60.

Sadavarte K, Mahobe H, Saxena G. Microbial contamination of Indian currency in Bhopal. 2014, J Evol. Med. Dental Sci., 3:1379-84.

Singh DV, Thakur K, Goel KA. 2002. Microbiological surveillance of currency. Indian J Med Microbiol., 20: 53.

Singh S, Singh M, Tiwari M, Kumar S, Kumari P, Saxena S.2015. Indian currency uncovered with microbes retrieved from expected and unexpected transaction points. Int $J$ Med Public Health, 5:242-6.

Tagoe DNA, Adams L, Kangah VG. 2011. Antibiotic resistant bacterial contamination of the Ghanian Currency Note. A potential health problem. J. Microbiol. Biotech. Res., 1: 37-44.

Clinical and Laboratory Standards Institute. 2012.

\section{How to cite this article:}

Zarrin Afroz. 2018. Bacteriological Profile and Antimicrobial Susceptibility Pattern of Indian Currency Circulating in a Tertiary Care Hospital of South India. Int.J.Curr.Microbiol.App.Sci. 7(04): 1828-1834. doi: https://doi.org/10.20546/ijcmas.2018.704.207 